\title{
ATELECTASIAS EM PEDIATRIA: MECANISMOS, DIAGNÓSTICO E TRATAMENTO
}

\author{
Cíntia Johnston*, Werther Brunow de Carvalho \\ Trabalho realizado na Universidade Federal de São Paulo - Escola Paulista de Medicina, São Paulo, SP
}

*Correspondência:

Rua da Consolação, 3367

CJ 104

CEP 01416-001

São Paulo - SP

Tel: (1 1) 82320542

cintiajohnston@terra.com.br

\begin{abstract}
RESUMO
OBjetivo. Realizar uma revisão da literatura sobre os mecanismos, diagnóstico e tratamento das atelectasias pulmonares em pediatria.

Métodos. Efetuou-se uma pesquisa nos bancos de dados eletrônicos Medline e Scielo com os critérios de inclusão: artigos publicados entre os anos de 1960 e 2007, que abordassem a etiologia, fisiopatologia, consequeências funcionais, avaliação, prevenção, tratamento e complicações das atelectasias pulmonares em pediatria. Palavraschave: atelectasis, children, pediatrics, mucus plugs, chest physiotherapy; atelectasias, criança, pediatria, fisioterapia respiratória, secreção brônquica. Utilizou-se o escore de Sackett DL, 1989 para a classificação do nível de evidência dos artigos encontrados.

Resultados. Foram analisados 45 artigos sobre atelectasias pulmonares, sendo 17 artigos em pediatria. Dos artigos com pacientes pediátricos, 13 estudos com série de casos clínicos, três artigos de revisão da literatura e um relato de caso, demonstrando que poucos artigos abordando atelectasias em pediatria foram publicados no período da pesquisa e que o nível de evidência dos artigos existentes são $D$ e $E$.

Conclusõos. Poucos estudos clínicos foram realizados atualmente para identificar o tratamento mais eficaz para a resolução das atelectasias em pediatria. Embora a prática clínica tenha evoluído no tratamento das mesmas, com o aperfeiçoamento das técnicas de broncoscopia e da fisioterapia respiratória existe a necessidade da realização de estudos clínicos randomizados nesta área.
\end{abstract}

UnItermos: Atelectasias. Pediatria. Doenças respiratórias. Insuficiência respiratória. Fisioterapia respiratória.

\section{INTRODUÇão}

As atelectasias são alterações pulmonares freqüentes em unidades de cuidados intensivos $(\mathrm{UCl})^{\prime}$ e salas de recuperação póscirúrgicas, podendo determinar piora do quadro clínico da criança com predisposição à complicações infecciosas e necessidade de maior suporte ventilatório [oxigenoterapia, ventilação pulmonar mecânica (VPM) invasiva e/ou não-invasiva]. Elas devem ser prevenidas, reconhecidas e tratadas para se evitar as suas complicações e conseqüente morbidade.

Nesta revisão da literatura serão abordados a etiologia, fisiopatologia, conseqüências funcionais, avaliação, prevenção, tratamento e as complicações das atelectasias em pediatria.

\section{Etiologia}

Os principais tipos de atelectasias pulmonares podem ser explicados em quatro subdivisões (Tabela I), com as suas respectivas etiologias:

I - Atelectasias de reabsorção, decorrentes da obstrução brônquica por plugs de secreção ou por corpo estranho na luz do brônquio; alterações da parede do brônquio (ex: edema da mucosa, inflamaçãa, tumores ou espasmos da musculatura lisa); compressão extrínseca por tumores ou gânglios, ou por vasos sangüíneos dilatados ou anômalos.
2 - Atelectasias de relaxamento ou compressão, decorrentes da pressão local direta no parênquima pulmonar. Ocorre devido ao aumento cardíaco, tumores ou deslocamento de vísceras, como na hérnia diafragmática ou eventração do diafragma; ou devido a pressão intrapleural aumentada, ocasionada por transudato, exudato e/ou ar no espaço pleural.

3 - Tensão superficial do alvéolo alterada, decorrente da alteração no revestimento alveolar (déficit de surfactante).

4 - Redução da elasticidade ou da complacência do parênquima pulmonar, que impossibilita a manutenção adequada das capacidades e volumes pulmonares.

\section{Fisiopatologia}

Quando a via aérea está ocluída, o ar fica aprisionado e os gases são absorvidos pelo sangue que perfunde o local anatômico do colapso pulmonar. A taxa de absorção depende da solubilidade dos gases: ar atmosférico, o nitrogênio e o hélio são absorvidos em duas a três horas; o oxigênio $\left(\mathrm{O}_{2}\right)$ a $100 \%$ é absorvido em poucos minutos, ocasionando colapso rápido da unidade pulmonar em seis minutos². Por isso, a atelectasia é freqüente no período pós-operatório, no qual geralmente são utilizadas altas taxas de concentração de $\mathrm{O}_{2}$.

A atelectasia do perioperatório pode ocorrer devido a alta taxa de reabsorção dos gases, decorrente do aumento da fração inspirada de 
Tabelal - Tipos de atelectasias e correspondentes etiologias

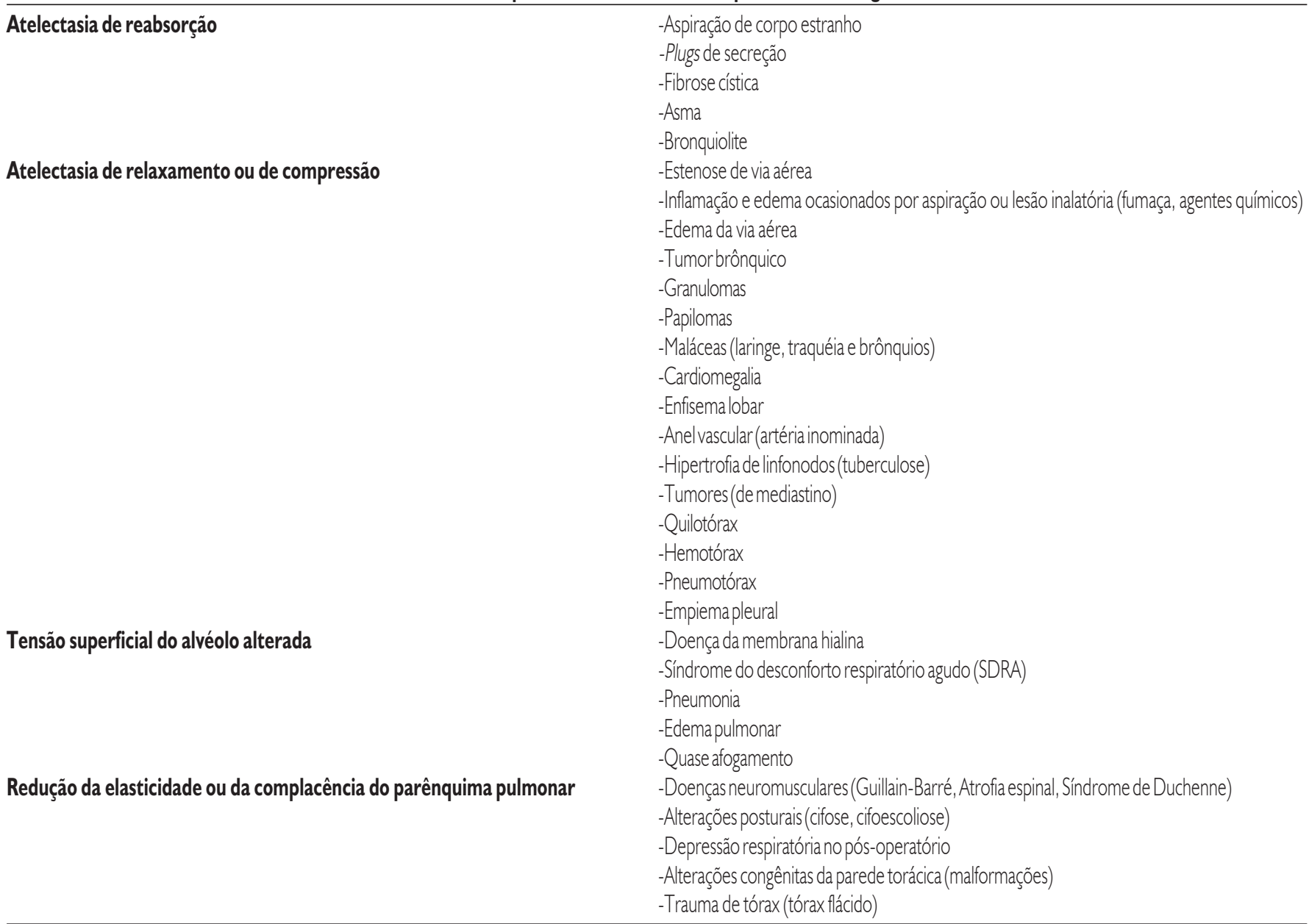

oxigênio e da redução da relação ventilação/perfusão (V/Q); por alteração do surfactante devido ao uso de agentes anestésicos, duração do ato cirúrgico e redução do volume corrente (VC); por compressão (tração) pulmonar decorrente da utilização de anestesia intravenosa ou inalatória; tempo de cirurgia; tipo de cirurgia; posicionamento do paciente no ato cirúrgico; obesidade; doença pulmonar prévia; idade entre outros fatores ${ }^{3}$.

A taxa e a extensão do colapso são modificados pela ventilação colateral através dos poros intra-alveolares (poros de Kohn) e pelas comunicações bronquíolos alveolares (canais de Lambert). Ambas estruturas apresentam menor desenvolvimento durante os primeiros meses de vida, por isso a atelectasia é mais freqüente em lactentes4.

A atelectasia pode ocorrer nas doenças com alterações de surfactante, pois ele apresenta uma ação direta na superfície de tensão alveolar, com conseqüente alteração do volume pulmonar. A propriedade de variação da tensão de superfície é atribuída ao componente fosfolipídico (fosfatidilcolina) do surfactante, mas outros lipídeos, pelo menos quatro proteínas ( $, B, C$ e D) associadas ao surfactante e o cálcio, estão presentes no surfactante natural e são necessários para a função pulmonar normal $\left.\right|^{5}$.
O pulmão contém fibras musculares lisas, envolvidas por fibras elásticas na porção distal da via aérea, incluindo os sacos alveolares. Este elemento mioelástico mantém o estado de contração do tecido pulmonar. A tendência ao colapso pulmonar é balanceada, na capacidade residual funcional (CRF), pela tendência da parede torácica de expandir, explicando por que o colapso pulmonar acompanha o pneumotórax, mesmo quando o sistema surfactante está intacto.

Nos pulmões normais, a insuflação alveolar e a perfusão vascular estão associadas com um baixo estresse e não são lesivos. Duas barreiras separadas formam a barreira alvéolo-capilar, o endotélio microvascular e o epitélio alveolar. Quando ocorre a atelectasia, a insuflação e a desinsuflação alveolar podem ser heterogêneas, resultando em estresse da via aérea, ocasionando lesão epitelial ${ }^{3}$.

Com a compressão dos vasos sangüíneos, a perfusão pode ser traumática e o fluxo poderá induzir a ruptura do endotélio microvascular. Tanto a lesão endotelial como a epitelial podem iniciar ou propagar a lesão pulmonar. Um colapso simples do alvéolo, com o tempo, poderá ocasionar uma reação inflamatória e os pulmões derrecrutados causarão lesão e perda da integridade epitelial, com lesão das células alveolares tipos 1 e 2. A lesão das células tipo 2 altera 
o transporte normal de fluídos pelo epitélio, modificando a remoção de fluído do edema do espaço alveolar e, adicionalmente ao colapso, os pulmões derrecrutados se tornam cheios de fluídos. Os neutrófilos aderem-se ao endotélio capilar lesado e migram através do interstício para os espaços alveolares. Nos espaços alveolares, os macrófagos secretam citocinas, interleucinas (IL-1,-6,-8 e - | 0 ) e fator de necrose tumoral alfa (TNF-alfa), que agem no local para estimular a quimiotaxia e ativar os neutrófilos. A IL-I pode estimular a produção de matriz extracelular pelos fibroblastos. Os neutrófilos podem liberar substâncias oxidantes, proteases, leucotrienos e outras moléculas próinflamatórias como o fator ativador de plaquetas ${ }^{6}$.

\section{Consequências funcionais}

A atelectasia está associada com conseqüências funcionais: alteração da oxigenação, redução da complacência pulmonar, aumento da resistência vascular pulmonar, hiperexpansão de unidades alveolares adjacentes, edema pulmonar após a reexpansão e lesão pulmonar?

Após o colapso de um segmento ou lobo pulmonar, a ventilação do alvéolo diminui, enquanto a perfusão poderá estar levemente diminuída, resultando em uma área com baixa relação $\mathrm{V} / \mathrm{Q}$. Se a região obstruída for grande, a hipoxemia poderá resultar do aumento da mistura venosa pelo shunt intrapulmonar ${ }^{3}$.

A redução da complacência pulmonar é resultado da diminuiç̧ão dos volumes pulmonares, decorrente do início de um ciclo ventilatório com uma CRF menor. Determinando um maior gasto de energia, com alteração da pressão transpulmonar, resultando em VC menor ${ }^{3}$. A resistência vascular pulmonar é mínima na CRF, o aumento do volume pulmonar resulta em compressão alveolar, decorrente do estiramento do tecido pulmonar. A queda do volume abaixo da CRF resulta em compressão dos vasos extra-alveolares. A hipóxia no local da atelectasia é um dos mecanismos que aumenta a resistência vascular pulmonar, devido a redução da tensão de $\mathrm{O}$, alveolar e venosa mista ${ }^{2}$. Outro efeito do segmento colapsado é a distensão do alvéolo adjacente não obstruído. Esta hiperdistensão compensatória pode ser proeminente no raio-x de tórax e pequenas áreas colapsadas podem não ser observadas ao raio-x ou evidentes na avaliação clínica. Se a hiperinsuflação for uma observação proeminente no raio-x, pode ser difícil determinar se a alteração primária é a atelectasia ou o enfisema, especialmente em recém-nascidos, nos quais as malformações congênitas com hipertransparência pulmonar são possíveis.

\section{Avaliação}

Os sinais e sintomas da obstrução brônquica variam com a extensão do colapso pulmonar. Em geral, a atelectasia que ocorre durante a evolução da tuberculose, asma,ou infecções como a bronquiolite, bronquite e broncopneumonia não ocasionam alteração no quadro clínico, exceto quando a aérea obstruída é extensa. Os sinais e sintomas, taquipnéia,tosse, estridor e dispnéia podem estar presentes devido à redução da areação do pulmão associada à obstrução ${ }^{7}$. Ocasionalmente, um chiado constante e localizado, a diminuição do murmúrio vesicular e a alteração da ressonância à percussão local sugerem atelectasia. Pode ser observada uma diminuição da expansão da caixa torácica, contração da musculatura e aproximação das costelas acima da área de atelectasia. No raio-x de tórax, o desvio do coração e do mediastino e a elevação do
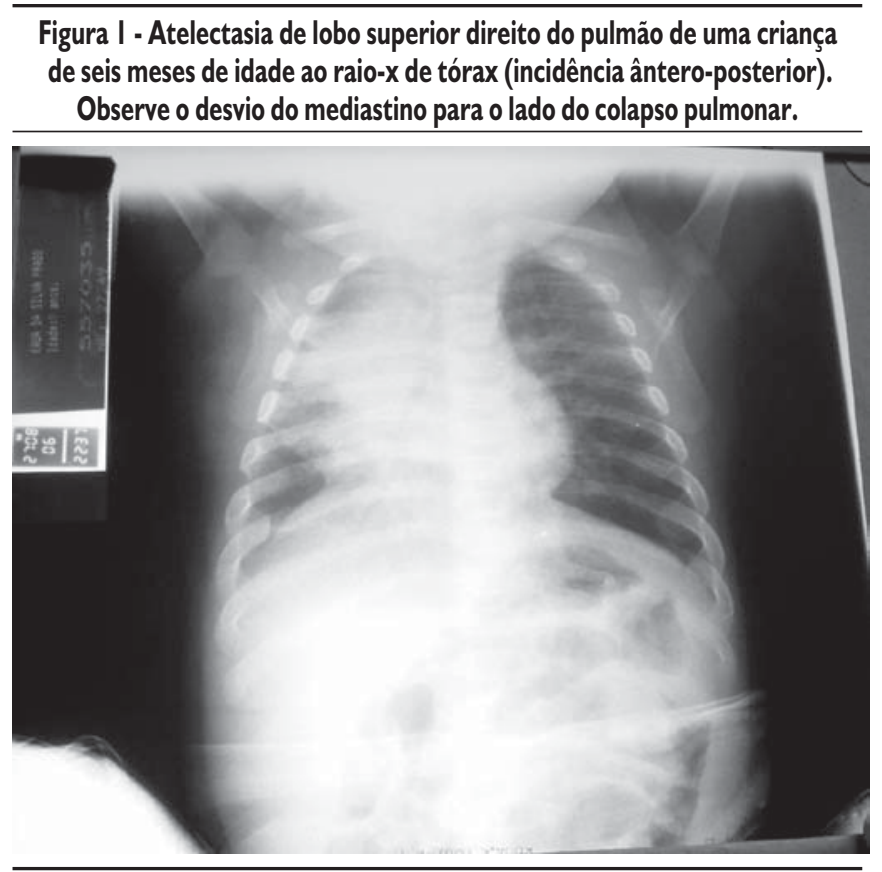

diafragma são observadas para o mesmo lado da atelectasia (Figura I) em colapsos extensos, mas em recém-nascidos e lactentes pode ser observado mesmo em colapsos menores ${ }^{8}$.

A atelectasia pulmonar é um sinal de doença, de forma isolada não é sugestiva de um diagnóstico específico. Quando identificada, o diagnóstico diferencial deve ser considerado e o exame mais útil e freqüentemente utilizado é o raio-x de tórax.

A atelectasia lobar ou segmentar é representada como opacificação do lobo ou segmento pulmonar? ${ }^{9}$. Geralmente, os sinais de atelectasia referem-se à perda de volume de ar/gás. O sinal mais direto é o deslocamento da fissura interlobar. Outros sinais de perda de volume, como o desvio do mediastino (Figura I) e aumento do hemidiafragma, são máximos perto do ponto da perda de volume. Pode haver hiperinsuflação compensatória, nos segmentos aerados remanescentes no lobo afetado, e a porção colapsada do pulmão demonstra o aumento da opacidade, sendo freqüentemente triangulares. Entretanto, a habilidade do raio-x de detectar a atelectasia que ocorre durante a anestesia ou durante $\mathrm{o}$ uso de VPM é menos acurada ${ }^{10,11}$.

A tomografia axial computadorizada (TAC) é o exame preferido para avaliar os pulmões, devido a sua resolução, velocidade e alta relação sinal/ruído. Nas imagens convencionais de TAC é possível medir os volumes pulmonares totais e regionais, a distribuição da areação pulmonar e o recrutamento em várias condições e intervenções clínicas' ${ }^{12}$. Na TAC de tórax, a atelectasia pode freqüentemente ser identificada pelos sinais de perda de volume, quando se utiliza o contraste, a atelectasia resulta em uma atenuação relativamente elevada do parênquima pulmonar, um achado útil para distinguir a atelectasia dos processos de consolidação (pneumonia), em que há uma atenuação relativamente baixa. A ressonância magnética (RM) permite a obtenção de imagens tridimensionais sem a utilização de irradiação 
ionizante. Embora a RM apresente várias vantagens (não é necessário o uso de contraste, possibilidade de múltiplas imagens planas) em relação à TAC, ela não oferece nenhuma vantagem no que se refere às atelectasias.

\section{Prevenção e tratamento}

Os fatores importantes para a prevenção ou para a reversão da atelectasia dependem de os pulmões serem ou não lesados. Os achados pré-clínicos do pulmão com atelectasia podem reduzir a propensão à lesão subseqüente ${ }^{13,14}$.

A atelectasia progressiva (e a alteração da oxigenação associada) pode ocorrer durante a ventilação constante se não for utilizada uma hiperinsuflação periódica. Este tipo de atelectasia é reversivel pela hiperinsuflação passiva (três insuflações sucessivas; $1^{\circ}{ }^{-}$com uma pressão de $10 \mathrm{cmH}_{2} \mathrm{O}$ durante 10 segundos; $2^{\circ}$ - com uma pressão de 30 $\mathrm{CmH}_{2} \mathrm{O}$ durante 15 segundos; $3^{\circ}$ - com uma pressão de $40 \mathrm{cmH}_{2} \mathrm{O}$ mantida por 15 segundos $)^{15}$. Os alvéolos colapsados são reabertos pelas ventilações profundas, com retorno da complacência pulmonar aos valores controles após as ventilações, que podem ser efetuadas com o aparelho de VPM ou com a hiperinsuflação manual com uma bolsa auto-inflável.

A aplicação de uma pressão expiratória final positiva (PEEP) de 10 $\mathrm{cmH}_{2} \mathrm{O}$ pode reabrir o tecido colapsado ${ }^{16,17}$. Entretanto, algumas atelectasias persistem, sendo necessário aumentar a PEEP para reexpandir essas unidades alveolares, mas a PEEP pode não ser a ideal, pois o shunt intrapulmonar não é diminuído e a oxigenação arterial pode não melhorar. A persistência do shunt pode ser explicada pela redistribuição do fluxo sangüíneo para as zonas dependentes do pulmão, quando se aumenta a pressão intratorácica, assim o pulmão com colapso residual recebe uma grande parte do fluxo sangüíneo pulmonar quando a PEEP é aplicada ${ }^{18}$. O aumento da pressão intratorácica pode diminuir o retorno venoso e o débito cardíaco. O pulmão pode recolapsar rapidamente após a descontinuação da PEEP, de forma que após um minuto de cessada a sua utilização, o colapso é tão grande quanto ao anterior à sua aplicação.

Várias estratégias ventilatórias podem produzir ou piorar a lesão pulmonar $^{19}$, como a utilização de VC elevados, altas taxas de pico de pressão e o colapso alveolar no final da expiração com a reabertura cíclica. A lesão pulmonar secundária à ventilação é atenuada se 0 volume no final da expiração for mantido pela administração da $P E{ }^{20,21}$. Adicionalmente, a ventilação com uma CRF baixa piora a lesão pulmonar, possivelmente pelo fechamento repetido das pequenas vias aéreas, a PEEP atenua esta lesão22. Portanto, o recrutamento do pulmão atelectásico reduz os efeitos lesivos da VPM.

O tratamento da atelectasia depende da causa, duração e da sua gravidade. Em pacientes hospitalizados, a atelectasia lobar, que ocorre após a cirurgia ou durante a VPM, deve ser tratada com fisioterapia respiratória e broncodilatadores. Tradicionalmente, o tratamento das atelectasias em UCl baseia-se na aspiração das vias aéreas, fisioterapia respiratória e broncoscopia quando necessário (persistência da atelectasia). Atualmente, tem sido adicionado a este tratamento a utilização das camas cinéticas e vibração mecânica, a terapêutica com agentes mucolíticos e a instilação ou a inalação de DNase recombinante humana. Aproximadamente $8 \%$ das crianças em VPM desenvolvem atelectasia pulmonar com aumento concomitante da morbidade e tempo de permanência hospitalarr ${ }^{23}$. Não existe padrão-ouro para o tratamento da atelectasia em pediatria.

O muco nos pacientes com fibrose cística (FC), bronquiectasias e bronquiolite pelo vírus sincicial respiratório contém uma quantidade significante de DNA extracelular dos leucócitos degenerados e dos debris epiteliais ${ }^{24}$. O DNA aumenta a viscosidade e a aderência das secreções pulmonares ${ }^{25}$. A utilização da DNase recombinante humana é efetiva para a reabertura das vias aéreas de pacientes com $\mathrm{FC}^{26}$. Nas infecções complicadas por atelectasia, as secreções brônquicas e plugs de muco apresentam alta concentração de DNA, de maneira que a DNase pode ser um tratamento efetivo nestas situações. Hendrikis et al, $2005^{27}$ utilizaram a DNase (aplicada por inalação ou instilação pela cânula traqueal, duas vezes ao dia até a melhora clínica) em pacientes pediátricos sem FC e com atelectasia, observando uma melhora clínica após duas horas e resolução das atelectasias após 24 hs.

A broncoscopia é moderadamente efetiva em remover as secreções e melhorar a reexpansão pulmonar (taxa de sucesso entre 19\% e 89\%), dependendo das características dos subgrupos de pacientes analisados ${ }^{28}$. Os subgrupos de pacientes com atelectasia lobar podem apresentar melhor resposta à broncoscopia do que aqueles com retenção de secreção ou atelectasia segmentar. Isso porque os pacientes com comprometimento lobar têm presumidamente grandes plugs centrais, que podem ser facilmente removidos pela broncoscopia. Adicionalmente, tem sido sugerido que o lavado broncoalveolar apresenta benefício adicional para o carreamento dos plugs mucosos distais.

Existem poucos estudos abordando o tratamento da atelectasia com fisioterapia respiratória em pediatria. Um estudo 29 identificou que a utilização da percussão manual e da drenagem postural não adiciona nenhuma eficácia para o tratamento da atelectasia aguda em relação à tosse e à respiração profunda. Em pacientes adultos ${ }^{30}$, quando comparados dois grupos (Grupo I = hiperinsuflação manual + aspiração das vias aéreas; Grupo 2 = vibração manual + drenagem postural), observou-se um aumento na taxa de resolução das atelectasias (8\% e 60\%), com melhores resultados no Grupo I. O estudo randomizado controlado ${ }^{31}$ com 37 pacientes adultos, comparando o tratamento com fisioterapia respiratória (sem suporte ventilatório: inspirações profundas durantes três minutos; ou intubados: múltiplas insuflações de IL a 2L com uma bolsa auto-inflável, de quatro em quatro horas; nebulização, aspiração e drenagem postural) com um grupo de pacientes submetidos à broncoscopia. Concluíram que não houve diferença na resolução da taxa de atelectasia entre os grupos após $24 \mathrm{~h}$ ou $48 \mathrm{~h}$ de realização dos procedimentos.

Embora a fisioterapia respiratória e a broncoscopia sejam os tratamentos freqüentemente utilizados para a atelectasia, existem poucos estudos randomizados e controlados abordando estes métodos de tratamento. Entretanto, na clínica diária de fisioterapeutas pediátricos, há uma tendência a não utilização da percussão manual (tapotagem) e, a utilização freqüente da hiperinsuflação manual associada à PEEP; a hiperinsuflação manual associada ao direcionamento de fluxo; hiperinsuflação com a utilização do push do aparelho de VPM associada ao direcionamento de fluxo; recrutamento alveolar; a utilização da VPM não-invasiva com pressão positiva e a utilização de técnicas de inspirações profundas ativas. Também tem-se optado pela intervenção 
fisioterapêutica nos casos de atelectasia após a extubação $0^{31,33}$, principalmente quando é necessário a utilização de altas taxas de $\mathrm{O}_{2}{ }_{2}^{34}$.

Um estudo experimenta ${ }^{15}$ demonstrou que a técnica de fisioterapia respiratória de compressão torácica (na fase expiratória da ventilação) com descompressão brusca ao início da fase inspiratória não contribuiu para a reexpansão das unidades alveolares colapsadas de ratos sedados e submetidos à VPM, pois não modificou a oxigenação $\left(\mathrm{PaO}_{2} / \mathrm{FiO}_{2}\right)$, a complacência pulmonar e a $\mathrm{PaCO}_{2}$.

Para reexpandir áreas com atelectasia é necessário que os plugs de muco sejam removidos e que ocorra aumento da pressão transpulmonara,37,38. A técnica de compressão e descompressão torácica brusca auxilia na remoção do muco, pois aumenta o tempo expiratório, mas não contribui para a reexpansão pulmonar em pediatria, pois para que isto ocorra é necessária a utilização de altas pressões inspiratórias ${ }^{36}$. Desta forma, a compressão torácica bilateral é contra-indicada para recém-nascidos e lactentes, pois ao comprimir-se bilateralmente o tórax conseqüentemente reduz-se temporariamente o recuo elástico pulmonar e, como nesta faixa etária a criança apresenta menor VC, ela não é capaz de gerar espontaneamente um fluxo inspiratório suficiente para retornar ao VC basal, o que provavelmente poderá predispor ao colapso de unidades alveolares ${ }^{39}$. Nesta faixa etária estão indicadas as técnicas de aumento do fluxo expiratório (AFE) sem compressão tóracoabdominal, associada ou não à vibração manual ${ }^{39-43}$.

A VPM não-invasiva pode ser utilizada para o tratamento da atelectasia. Geralmente, tem-se optado pela utilização do modo ventilatório com pressão positiva em dois níveis (BiPAP). Em pacientes adultos no pós-operatório de cirurgia bariátrica ${ }^{44}$, identificou-se que a aplicação de BiPAP (pressão inspiratória positiva - IPAP de I $2 \mathrm{cmH}_{2} \mathrm{O}$ e pressão expiratória positiva - EPAP de $4 \mathrm{cmH}_{2} \mathrm{O}$ ) melhorou a capacidade vital forçada e o volume expiratório forçado no primeiro segundo após 24h da sua utilização. Em pediatria, não existem estudos avaliando a utilização da VPM não-invasiva para o tratamento das atelectasias pulmonares. Porém, na prática clínica, esta modalidade tem sido freqüentemente utilizada de forma intermitente.

\section{Complicações e prognóstico}

As atelectasias aguda e subaguda são geralmente benignas e respondem à terapêutica. Uma evolução ruim é provável quando a causa subjacente à atelectasia (trauma de tórax) é ameaçadora à vida ou quando a área perdida de troca gasosa não é reconhecida, ou não responde ao tratamento (aspiração de corpo estranho, plug de muco intra operatório, que pode ocasionar colapso total do pulmão).

O edema agudo pulmonar pode ocorrer após a reexpansão de uma área grande de atelectasia, após o tratamento de grandes pneumotórax e derrames pleurais ${ }^{45,46}$. Geralmente, o edema acomete o pulmão reexpandido e ocorre entre uma e 36 horas após o recrutamento alveolar. O mecanismo fisiopatológico que ocasiona este edema não é totalmente esclarecido na literatura.

É importante a manutenção dos volumes pulmonares para prevenir a lesão pulmonar. O colapso repetido do pulmão ocasiona uma ativação dos neutrófilos em pulmões previamente lesados ${ }^{47,48}$. A presença de atelectasia contribui para o efeito inflamatório dos pulmões.

\section{Conflito de interesse: não há}

\section{SUMMARY}

Atelectasis: MeChanISMS, Diagnosis AND tReATMENT IN THE PEDIATRIC PATIENT

OBJECTIVE. To review the literature about mechanisms, diagnosis and treatment of atelectasis in the pediatric patient.

MetHODS. An electronic data search was carried out in Medline and Scielo using the following inclusion criteria for articles published between 1960 and 2007 about: atelectasis etiology, physiopathology, functional consequences, evaluation, prevention, treatment and complications, in pediatrics. The used key words were atelectasis, children, pediatrics, mucus plugs, chest physiotherapy;

RESULTS. 45 pulmonary atelectasis articles were analyzed, 17 of them in pediatrics. Ofthe pediatric, 13 were case series, 3 literature reviews and one a case report. This demonstrates that there were few articles on atelectasis during the reviewed period and that these articles were at the $D$ and E evidence level.

CONCLUSION. No clinical trials were performed to identify s the most efficient treatment for atelectasis in the pediatric patient. Although clinical practice for treatment of atelectasis has evolved, mostly due to improvement of bronchoscopy and chest physiotherapytechniques, there is still a need to perform randomized clinical trials to address treatment of atelectasis in the pediatric patient. [Rev Assoc Med Bras 2008; 54(5): 455-60]

KEY wORDs: Atelectasis, pediatrics. Respiratory diseases. Respiratory failure. Chest physiotherapy.

\section{REFERÊNCIAS}

I. Thomas K, Habibi P, Britto J, Owens CM. Distribution and pathophysiology of acute lobar collapse in the pediatric intensive care unit. Crit Care Med. 1999;27:1677-9.

2. Benumof JL. Mechanism of decreased blood flow to atelectatic lung. J Appl Physiol. 1979:46: 1047-8.

3. Duggan M, Kavanagh BP. Atelectasis in the perioperative patient. Curr Opin Anaesthesiol. 2007;20:37-42.

4. Hazinski TA. Atelectasis. In: Chermick and Boat, editors. Kendig's disorders of respiratory tract in children. Philadelphia: WB Saunders; |998. p.634-41.

5. Pinheiro CTS, Carvalho WB (eds). Programa de Atualização em Medicina Intensiva. Ciclo 4,Módulo 3. p.9-40. Editora Artmed: Porto Alegre, 2007.

6. Tremblay L, Valenza F, Ribeiro SP, Li J, Slutsky AS. Injurious ventilatory strategies increase cytokines and c-fos $\mathrm{m}$-RNA expression in an isolated rat lung model. J Clin Invest. 1997;99:944-52.

7. Peroni DG, Boner AL. Atelectasis: mechanisms, diagnosis and management. Paediatr Respir Rev. 2000; 1 :274-8.

8. Swischhuk LE, John SD. Differencial diagnosis in pediatric radiology. Baltimore: Williams and Wilkins, 1995;64-78.

9. Ashizawa K, Hayashi K, Aso N, Minami K. Lobar atelectasis diagnostic pitfalls on chest radiography. Br J Radiol. 200 I,74:89-97.

10. Prys-Roberts C, Nunn JF, Dobson RH, Robinson RH, Greenbaum R, Harris RS.

I1. Radiologically undetectable pulmonary collapse in the supine position. Lancet. 1967;2:399-40I.

12. Magnusson L, Spahn S. New concepts of atelectasis during general anaesthesia. Br J Anaesth. 2003;91:61-72.

13. Lundquist $H$, Hedenstierna G, Strandberg A, Tokics L, Brismar B. Assessment of dependent lung densities in man during general anaesthesia. Acta Radiol. 1995;36:626-32. 
14. DeCampos KN, Keshavjee S, Slutsky AS, Liu M. Alveolar recruitment prevents rapid reperfusion-induced injury of lung transplants. J Heart Lung Transplant. 1999; | 8: 1096-102.

15. Hamvas A, Park CK, Palazzo R, Liptay M, Cooper J, Schuster DP. Modifying pulmonary ischemia-reperfusion injury by altering ventilatory strategies during Ischemia. J Appl Physiol. 1992;73:21 I2-9.

16. Bendixen HH, Hedley-Whyte J, Laver MB. Impaired oxygenation in surgical patients during general anesthesia with controlled ventilation. $\mathrm{N}$ Engl J Med. 1963;269:99|-6.

17. Hachenberg T, Lundquist H, Tokics L. Analysis of lung density by computed tomography before and during general anaesthesia. Acta Anaesthesiol Scand. 1993;37:549-55.

18. Tokics L, Hedenstierna G, Strandberg A. Lung collapse and gas exchange during general anesthesia: effects of spontaneous breathing, muscle paralysis, and positive endexpiratory pressure. Anesthesiology. 1987;66: 157-67.

19. West JB, Dolley CT, Naimark A. Distribution of blood ow in isolated lung: relations to vascular and alveolar pressure. J Appl Physiol. 1964; 1 9: 13-24.

20. Parker JC, Hernandez LA, Peevy KJ. Mechanisms of ventilator-induced lung injury. Crit Care Med. 1993;21: I3 I-43.

21. Chiumello D, Pristine G, Slutsky AS. Mechanical ventilation affects local and systemic cytokines in an animal model of acute respiratory distress syndrome. Am J Respir Crit Care Med. 1999; 160: 109-16.

22. Dreyfuss D, Soler P, Basset G, Saumon G. High inflation pressure pulmonary edema: respective effects of high airway pressure, high tidal volume, and positive end-expiratory pressure. Am Rev Respir Dis. 1988; 137: 1 159-64.

23. Muscedere JG, Mullen JB, Gan K, Slutsky AS. Tidal ventilation at low airway pressures can augment lung injury. Am J Respir Crit Care Med. 1994; | 49: | 327-34.

24. Rivera R, Tibballs J. Complications of endotracheal intubation and mechanical ventilation in infants and children. Crit Care Med. 1992,20:193-9.

25. Nasr SZ, Strouse PJ, Soskolne E. Efficacy of recombinant human deoxyribonuclease I in the hospital management of respiratory syncytial virus bronchiolitis. Chest. 200 I, 120:203-8.

26. Puchelle E, Zahm JM, de Bentzmann S, Grosskopf C, Shak S, Mougel D, et al. Effects of rhDNase on purulent airway secretions in chronic bronchitis. Eur Respir.J 1996;9:765-9.

27. Quan JM, Tiddens HA, Sy JP, McKenzie SG, Montgomery MD, Robinson PJ, et al. Pulmozyme Early Intervention Trial Study Group: A two-year randomized, placebo-controlled trial of dornase alfa in young patients with cystic fibrosis with mild lung function abnormalities. J Pediatr. 2001, 139:813-20.

28. Hendriks T, de Hoog M, Lequin MH, Devos AS, Merkus PJ. DNase and atelectasis in non-cystic fibrosis pediatric patients. Critical Care. 2005; 9:R35I-R6

29. Kreider ME, Lipson DA. Bronchoscopy for atelectasis in the ICU. Chest 2003; 124:344-50.

30. Johnson N, Marini JJ, Pierson DJ. Acute lobar atelectasis: effect of chest percussion and postural drainage (CPPD) on resolution. Am Rev Respir Dis. 1 987: 1 35:A433.

31. Stiller K, Geake T, Taylor J, Grant R, Hall B. Acute lobar atelectasis: a comparison of two chest physiotherapy regimens. Chest. 1990;98: 1336-40.

32. Al-Alaiyan S, Dyer D, Khan B. Chest physiotherapy and post-extubation atelectasis in infants. Pediatr Pulmonol. 1996;21:227-30.
33. Bloomfield FH, Teele RL, Voss M, Knight DB, Harding JE. The role of neonatal chest physiotherapy in preventing postextubation atelectasis. J Pediatr. 1998; |33:269-7|.

34. Marini JJ, Pierson DJ, Hudson LD. Acute lobar atelectasis: a prospective comparison of fiberoptic bronchoscopy and respiratory therapy. Am Rev Respir Dis. 1979; | 19:97| I-8.

35. Benoît Z, Wicky S, Fischer JF, Frascarolo P, Chapuis C, Spahn DR, et al. The effect of increased $\mathrm{FIO}_{2}$ before tracheal extubation on postoperative atelectasis. Anesth Analg. 2002;95: 1777-81.

36. Unoki $\mathrm{T}$, Mizutani $\mathrm{T}$, Toyooka $\mathrm{H}$. Effects of expiratory rib cage compression and/or prone position on oxygenation and ventilation in mechanically ventilated rabbits with induced atelectasis. Respir Care. 2003:48:754-62.

37. Tsao TC, Tsai YH, Lan RS, Shieh WB, Lee CH. Treatment for collapsed lung in critically ill patients: selective intrabronchial air insufflation using the fiberoptic bronchoscope. Chest. 1990;97:35-438.

38. Duggan MD, Kavanagh BP. Pulmonary atelectasis. Anesthesiology. 2005; | 02:838-54.

39. Govaere E, Van Raemdonck D, Devlieger H, Smet MH, Verbeken E, Proesmans $\mathrm{M}$, et al. Massive lung collapse with partial resolution after several years: a case report. BMC Pediatrics. 2005;5:39.

40. Freitag L, Long WM, Kim CS, Wanner A. Removal of excessive bronchial secretions by asymmetric high-frequency oscillations. J Appl Physiol. 1989:67:6|4-9.

41. Henschen M, Stocks J, Hoo AF, Dixon P. Analysis of forced expiratory maneuvers from raised lung volumes in preterm infants. J Appl Physiol. 1998;85: 1989-97.

42. Almeida CC, Ribeiro JD, Almeida-Júnior AA, Zeferino AM. Effect of expiratory flow increase technique on pulmonary function of infants on mechanical ventilation. Physiother Res Int. 2005; 10:2 I3-21.

43. Manfred M, Eber E, Weinhandl E. A Comparison of the Tidal and the Raised Volume Rapid Thoracoabdominal Compression Technique. Am J Respir Crit Care Med. 2000; 161:763-8.

44. Platzker AC, Colin AA, Chen XC, Hiatt P, Hunter J, Koumbourlis AC, et al. Thoracoabdominal compression and respiratory system compliance in HIV-infected Infants. Am J Respir Crit Care Méd. 2000, 161: | 567-71.

45. Joris JL, Sottiaux TM, Chiche JD. Effect of bi-level positive airway pressure (BiPAP) nasal ventilation on the postoperative pulmonary restrictive syndrome in obese patients undergoing gastroplasty. Chest. | 997; | | |:665-70.

46. Mahfood S, Hix WR, Aaron BL, Blaes P, Watson DC. Reexpansion pulmonary edema. Ann Thorac Surg. 1988;45:340.

47. Greene R. Acute lobar collapse: adults and infants differ in important ways. Crit Care Med. 1999:27:1677-9.

48. Imai Y, Kawano T, Iwamoto S, Nakagawa S, Takata M, Miyasaka K. Intratracheal anti-tumor necrosis factor-alpha antibody attenuates ventilator-induced lung injury in rabbits. J Appl Physiol. 1999;87:5 I0-5.

49. Kawano T, Mori S, Cybulsky M, Burger R, Ballin A, Cutz E, et al. Effect of granulocyte depletion in a ventilated surfactant-depleted lung. J Appl Physiol. 1987;62:27-33.

Artigo recebido: 1 1/06/07 Aceito para publicação: 24/03/08 